\title{
Measuring compliance of package inserts in the Emirate of Abu Dhabi-UAE
}

\author{
Hadaya Gharibyar ${ }^{1^{*}}$, Yasser Sharif ${ }^{2}$, Sherif El Ghandour ${ }^{1}$, Maysoon Saffarini $^{1}$, Bashir Aden $^{1}$ \\ ${ }^{1}$ Health Authority_Abu Dhabi, Abu Dhabi, UAE; *Corresponding Author: hgharibyar@haad.ae \\ ${ }^{2}$ Section Head Medication \& Medical Products Safety, Health Authority—Abu Dhabi, Abu Dhabi, UAE
}

Received 7 February 2013; revised 12 March 2013; accepted 21 March 2013

Copyright (c) 2013 Hadaya Gharibyar et al. This is an open access article distributed under the Creative Commons Attribution License, which permits unrestricted use, distribution, and reproduction in any medium, provided the original work is properly cited.

\begin{abstract}
Purpose: The aim of this study was to examine if package inserts (PIs) supplied with prescribed medications in the Emirate of Abu Dhabi contain all relevant information to the safe and appropriate use of these medications. Methods: Sixty seven PIs for prescription-only medications were evaluated against a set of safety criteria published from the Ministry of Health. Results: Analyzed PIs showed many deficiencies with regard to the Ministry of Health (MOH) Investigation New Drug Application (INDA) requirements. Particularly of concern were side effects, warnings, use in pregnancy, lactation, and the storage conditions for the product. Conclusions: This study indicated that information relevant to the safe and appropriate use of medications was not uniformly mentioned in the PIs analyzed. To avoid medication errors due to deficits in the current PIs, we recommend regulatory oversight and regulator audits from pharmaceutical company, followed by enhancement of regulations requiring companies to also create patient information leaflets.
\end{abstract}

Keywords: Drug Labeling; Generic Medications; Adverse Reactions; Drug Safety;

Pharmacoepidemiology; Drug Information; Abu

Dhabi; UAE; Package Inserts

\section{INTRODUCTION}

It has long been established that package inserts (PIs) (also referred to as product information, prescribing information, or Physicians' Desk Reference [PDR] listing) are one of the most frequently used sources of drug information by healthcare professionals [1,2]. Having reli- able and complete information in package inserts is essential for effective and safe use of medications. Low quality and incomplete information could have a potentially negative impact on patient healthcare [3]. This impact was observed through drug utilization evaluations conducted through the Health Authority-Abu Dhabi Pharmacy \& Therapeutics Committee. For instance, physicians quite often prescribed for off-labeled indications simply because the package inserts did not contain sufficient information about all the labeled indications. It has also been observed in another study that physician utilization of package inserts which contained incomplete or absent information, with regard to side effects, drug interactions, warnings and precautions, use in lactation and pregnancy, may contribute to an increase in emergency room visits [3].

Package inserts are prepared by pharmaceutical companies and approved by health regulating bodies. Drug products that are approved for use in the United Arab Emirates originate mainly from multinational companies from the USA, Europe, and some local manufacturers. Package insert requirement in the USA and Europe vary only slightly in that in the USA for only certain therapeutic categories, patient oriented information, in addition to healthcare oriented information, is included, while in Europe patient information leaflets (also known as PIL) are mandatory for all drug products [3-5].

In the United Arab Emirates (UAE) (a country with seven Emirates or States), healthcare is regulated by the Federal government body known as the Ministry of Health $(\mathrm{MOH})$, which only requires that the package insert be oriented only towards healthcare professionals. The $\mathrm{MOH}$ has specific criteria in the Investigational New Drug Application (INDA) for the minimum information necessary to be contained in package inserts. Companies with new products are all asked to submit package inserts that meet these requirements. The clinically oriented requirements are similar to the USA and Europe. This is not 
surprising as not only do most drug products in the UAE come from Europe and USA but also the fact that in Europe and USA, substantial regulatory efforts have been made to improve written drug information, including package inserts [6,7].

Studies in the UAE have shown that package inserts are a major source of drug information for healthcare professionals [8] as well as a major marketing tool by pharmaceutical companies [9]. Studies have also shown that pharmaceutical companies in the UAE were less than compliant with regulations and international standards when disseminating their other promotional drug information material such as brochures and pamphlets [9].

The Health Authority-Abu Dhabi (HAAD) is the local government branch responsible for regulating the healthcare industry and developing the health policy for the Emirate of Abu Dhabi. Package inserts which are approved by the $\mathrm{MOH}$ are included with drug products that are in circulation in Abu Dhabi. The Poison \& Drug Information Center (PDIC) wanted to ensure that these package inserts complied with the MOH INDA requirements. The aim of this study was to assess the quality and completeness of package inserts approved for use in the Emirate of Abu Dhabi utilizing the MOH INDA criteria.

\section{METHODS}

Brand name pharmaceutical companies, whose products were the most commonly prescribed for the top ten diseases (based on HAAD 2011 statistics report [10] in Abu Dhabi (Table 1), was selected for the study, in addition to local manufacturers, to provide their package inserts. For this we gathered a small convenience sampling of 67 package-inserts from different drug manufacturers during November 1 through 15, 2012.

The package inserts were evaluated using the $\mathrm{MOH}$ INDA requirements for the minimum information necessary to be contained in package inserts. Evaluation was based on whether they contained the headings required per the INDA criteria for 14 clinically important parameters (Table 2) [11]. Compliance was defined as meeting all 14 of the requirements. To check for completeness, the package inserts were then compared against the same product in the originator country. Comparator package inserts were obtained from respective international regulatory agency repositories. Descriptive and frequency statistical analysis was performed using CDC EpiInfo $\left(7^{\mathrm{TM}}\right)$.

\section{RESULTS}

Of the total $(n=67)$ package inserts were examined, 48 (71.6\%) were brand name and 19 (28.4\%) were from local generic manufacturers. Only 35 (52\%) (PIs) contained all relevant clinical information, while 32 (48\%)
Table 1. Distribution of top $10 \mathrm{~d}$ diseases prescribed by drugs Abu Dhabi, 2010.

\begin{tabular}{cc}
\hline 1. Diabetes mellitus & 2. Respiratory infections \\
3. Signs and symptoms, ill defined & 4. Cardiovascular disease \\
5. Musculoskeletal disease & 6. Endocrine disorders \\
7. Respiratory disease & 8. Digestive diseases \\
8. Skin diseases & 10. Genitourinary diseases \\
\hline
\end{tabular}

Table 2. Package inserts safety criteria by MOH INDA.

\begin{tabular}{cc} 
1. Description & 2. Clinical Pharmacology \\
3. Indications & 4. Dosage \\
5. Administration & 6. Contraindications \\
7. Side effects & 8. Drug interactions \\
9. Pregnancy & 10. Lactation \\
11. Warnings & 12. Precautions \\
13. Over dosage & 14. Storage conditions \\
\hline
\end{tabular}

did not complied with the MOH INDA requirements (Figure 1). A summary of drug class and distribution of country of origin appear in Table 3.

The quality of package inserts information was poor in drugs coming from regional manufacturers versus those drugs coming from North America or Europe.

Number of package inserts containing required patient safety and physician information was greater for brand name 34 (70.8\%) compared to $1(5.3 \%)$ generic drug names.

\section{DISCUSSION}

This study demonstrates that the drug package inserts analyzed only 35 (52.2\%) out 67 contain clinically relevant information that is required by the Ministry of Health $(\mathrm{MOH})$, while 32 (47.8\%) failed in one or more of the 14 parameters evaluated. However, it's important to note that the requirements for package inserts in the USA and Europe do vary in that in the USA, patient information is only required for certain products but in Europe it is required for all products [3-5].

In the UAE, package inserts are only required to be oriented towards healthcare professionals as defined by the MOH INDA criteria. Since products in the UAE originate from Europe, USA, Canada, and Japan, it was not surprising when analysis revealed that some of the prescribing information could be defined as a package insert intended for healthcare professionals, others for patients, and others a combination of the two.

Furthermore, for brand name companies, some products that were patient oriented simply could not qualify in many of the 14 criteria. For products that were healthcare professional oriented, the most common subject 


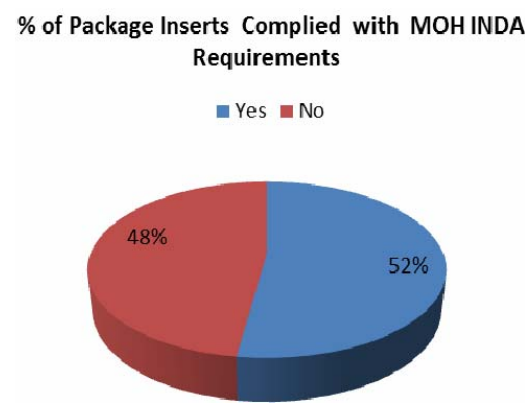

Figure 1. Percentage of packages inserts complied with MOH INDA requirements.

Table 3. Distribution of package inserts by class and country of origin of the drugs.

\begin{tabular}{|c|c|c|}
\hline Variable & Frequency & $\%$ \\
\hline \multicolumn{3}{|c|}{ Drug Class } \\
\hline Brand & 48 & $71.6 \%$ \\
\hline Generic & 19 & $28.4 \%$ \\
\hline Total & 67 & $100 \%$ \\
\hline \multicolumn{3}{|c|}{ Drug Country of Origin } \\
\hline USA & 28 & $41.8 \%$ \\
\hline UAE & 19 & $28.4 \%$ \\
\hline Sweden & 7 & $10.4 \%$ \\
\hline France & 5 & $7.5 \%$ \\
\hline UK & 4 & $6.0 \%$ \\
\hline Japan & 1 & $1.5 \%$ \\
\hline Germany & 1 & $1.5 \%$ \\
\hline Canada & 1 & $1.5 \%$ \\
\hline Belgium & 1 & $1.5 \%$ \\
\hline Total & 67 & $100 \%$ \\
\hline
\end{tabular}

heading not included in the package insert was the storage conditions for the product. Deficiencies for generic manufacturers tended to be across the board for all 14 of the defined criteria in not only patient oriented package inserts but also healthcare professionals ones as well. Information about over dosages, side effects, warnings, precautions, use in pregnancy and lactation, use in pediatric patients, and use in the elderly were quite often absent or deficient when compared against the brand name package insert of the originator country.

This finding is similar to the results of a study in a neighboring country that found limited and incomplete information when local generic package inserts were compared with their counterparts marketed in the USA $[12,13]$. Moreover, some of the package inserts did contain information with regard to side effects, warnings, use in pregnancy and lactation, it was severely lacking when compared against the package insert from the originator country. Other package inserts from multinational companies did not include all of the safety information found in the originator country, particularly for patient oriented package inserts.

Insufficient safety information may lead to avoidable adverse events resulting in emergency rooms visits. This was observed was observed through drug utilization evaluations conducted through the Health AuthorityAbu Dhabi Pharmacy \& Therapeutics Committee. This is supported by a study in the USA which showed that the presence of labeling differences (or deficiencies) may complicate physician practices raising the possibility that important safety information may be missed [14].

The results of this study show that compliance with the requirements set forth by the $\mathrm{MOH}$ via the INDA criteria is seriously in question. Additionally, a lack of regulatory compliance post the initial approval of the package inserts is essential to ensure compliance. Having incomplete or absent information in any of the clinically relevant parameters can impact physician prescribing patters but also has the potential to impact patient safe and effective use of medications.

\section{CONCLUSION}

This study indicates that package inserts that accompany drug products in the UAE have deficiencies with regard to the MOH INDA clinically relevant criteria, which could have an impact on patient health. To ensure the effective and safe use of medications, regulatory oversight and regulator audits are necessary to ensure continuous compliance, in addition to the voluntary compliance, which will be facilitated by the HAAD PDIC, by pharmaceutical companies.

\section{LIMITATIONS}

This study had limitations in that the number of package inserts that were evaluated was small. In some cases, products from certain countries i.e. 1 product showed the country to be $100 \%$ compliant with the requirements. Testing more products could have possibly shown less compliance. Second, the collection was based on the top ten disease states prescribing in the Emirate of Abu Dhabi, which was not random, which raised the possibility of selection bias. Despite these limitations, this study represents the first attempt to evaluate package inserts in the UAE. The findings in this study should stimulate further research particularly with regard to readability of 
these package inserts by patients, in addition to the full impact on low quality package inserts on patient health.

\section{REFERENCES}

[1] Joubert, P.H. and Skene, D. (1984) Attitudes of private medical practitioners towards package inserts and other drug information sources. South Africa Medical Journal, 66, 306-307.

[2] Fuchs, J., Hippius, M. and Schaefer, M. (2005) A survey of package inserts use by patients. Hospital Pharmacy Europe, 21, 29-31.

[3] Shivkar, Y.M. (2009) Clinical information in drug package inserts in India. Journal of Post Graduate Medicine, 55, 104-107. doi:10.4103/0022-3859.52840

[4] European Medicines Agency (2008) Guideline on the packaging information of medicinal products for human use.

http://ec.europa.eu/enterprise/pharmaceuticals/eudralex/v ol-2/c/bluebox_02_2008.pdf

[5] Food and Drug Administration (2006) Requirements on content and format of labeling for human prescription drug and biological products. Federal Register, 71, 39223997.

[6] Raynor, D.K., Svarstad, B., Knapp, P., et al. (2007) Consumer medication information in the United States, Europe, and Australia: A comparative evaluation. Journal of the American Pharmacists Association, 47, 717-724. doi:10.1331/JAPhA.2007.06141
[7] Raynor, D.K., Blenkinsopp, A., Knapp, P., et al. (2007) A systematic review of quantitative and qualitative research on the role and effectiveness of written information available to patients about individual medicines. Health $\mathrm{Te}$ chnology Assessment, 11, 1-160.

[8] Gharibyar, H., Sharif, Y., Al Qawasme, K. and Fahmy, S. (2012) Physicians' perception of drug information resources in the emirate of Abu Dhabi-UAE. Pharmacology \& Pharmacy, 52-56.

[9] Gharibyar, H. and Sharif, Y. (2012) Evaluation of pharmaceutical drug information brochures in the emirate of Abu Dhabi (United Arab Emirates). Journal of Pharmaceutical Health Services Research, 3, 57-62. doi:10.1111/j.1759-8893.2011.00071.x

[10] Health Authority_Abu Dhabi (2011) Health statistics. www.haad.ae/statistics

[11] Ministry of Health (2012) Investigation new drug application. www.moh.gov.ae/en/

[12] Bawazir, S.A., Al-Hassan, M.I., Al-Khamis, K.I., AbouAuda, H.S. and Gubara, O.A. (1991) Comparative study of Saudi-marketed products and US drug labeling. DICP, 25, 863-866.

[13] Al-Aqeel, S.A. (2012) Evaluation of medication package inserts in Saudi Arabia. Drug, Healthcare and Patient Safety, 4, 33-38. doi:10.2147/DHPS.S29402

[14] Duke, J., Friedlin, J. and Li, X. (2012) Consistency in the safety labeling of bioequivalent medications. Pharmacoepidemiology and Drug Safety, 22, 294-301. doi:10.1002/pds.3351 